\title{
L’éthique éditoriale dans l'intérêt de tous
}

Comme toute autre revue, Bois et Forêts des Tropiques est exposée à des pratiques non respectueuses de l'intégrité scientifique. Nous observons parfois chez des auteurs des écarts aux normes éthiques requises par l'édition. La pression éprouvée par les chercheurs pour plus publier, plutôt que mieux publier, n'y est sans doute pas tout à fait étrangère.

Et comme l'a récemment rappelé Pierre Léna, ancien président du Comité d'éthique du CNRS, le meilleur garant contre les manquements à l'éthique scientifique demeure la conscience du chercheur. Conscience individuelle, à l'évidence, mais aussi conscience collective. Aussi, à la pression de publication qui a désormais envahi le quotidien et la pensée des chercheurs, vient aujourd'hui s'opposer une réaction éthique de la part des institutions de recherche. Une réaction saine, nous semble-t-il, à laquelle notre revue s'associe entièrement.

Les fraudes manifestes que nous relevons dans Bois et Forêts des Tropiques sont heureusement rares et la plupart du temps, il s'agit plutôt de pratiques inappropriées. Ces pratiques n'en sont pas moins regrettables, d'autant qu'elles tendent à se multiplier mais aussi à se diversifier. Les manquements à une ligne de conduite intègre, tels que nous les observons dans les articles soumis à notre revue, présentent en effet un caractère largement multiforme.

Le manquement le plus courant procède d'un manque de clarté du protocole d'étude ou d'une négligence dans le traitement des données, qui se traduisent par des résultats incohérents, injustifiés ou erronés. La frontière entre l'erreur et la falsification s'avère toutefois ténue, ces deux notions étant en continuité l'une de l'autre. Généralement, les relecteurs repèrent toutefois très vite ce type de dérive, quand c'en est une.

Parfois aussi, des chercheurs ne respectent pas les principes élémentaires selon lesquels ils peuvent ou non apparaître légitimement comme coauteurs d'un manuscrit. Certains éditeurs n'hésitent pas à qualifier cette pratique de "complicité passive ». Les cas où, fournie par l'auteur correspondant, l'adresse électronique d'un coauteur s'avère non fonctionnelle, sont en soi particulièrement éloquents...

La soumission auprès de notre revue d'un texte déjà publié par les auteurs dans une autre revue reste heureusement une pratique peu commune. Ce qu'il convient d'appeler alors « autoplagiat » se produit néanmoins deux à trois fois par an. Généralement, ce type de pratique reste assez aisément détectable. Il l'est beaucoup moins lorsque le manuscrit soumis ne présente délibérément qu'une fraction de résultats déjà publiés ailleurs.

Une autre dérive relevant de la dissimulation consiste à soumettre le même manuscrit à plusieurs revues en même temps, ce qui conduit alors à des situations très inconfortables, voire de blocage, entre les revues et l'auteur correspondant, mais aussi entre les revues elles-mêmes.
Beaucoup plus fréquente, mais aussi certainement plus savoureuse, est l'approche préalable d'un auteur auprès de notre équipe éditoriale, selon la technique dite du " pied dans la porte ». Ceci consiste à nous inviter à accepter par avance un manuscrit qui n'a pas encore été soumis mais reste évoqué dans ses grandes lignes. Pour sympathique qu'elle soit parfois, cette approche n'est pas conforme aux fondamentaux de l'éthique éditoriale. Elle n'est donc pas davantage acceptable.

Il n'y a que des perdants dans la mise en jeu de l'ensemble de ces pratiques. Perte de temps, perte de crédibilité, y compris des institutions représentées par les auteurs indélicats, mais aussi manifestation d'une perte d'exigence scientifique. C'est donc au service de tous que nous sommes invités, au sein de notre équipe d'édition, à accroître notre vigilance. Ce type d'attention n'est pas des plus agréables, et nous ne voulons surtout pas entretenir, au sein de Bois et Forêts des Tropiques, un climat de suspicion à l'égard des auteurs. Ce serait sans aucun doute la pire des réactions parce qu'elle entretiendrait une forme de dénigrement de la recherche scientifique.

Alors que faire ? Il nous semble que la prévention et l'information auprès des auteurs, comme le vise cet éditorial, figurent parmi les meilleures attitudes possibles. L'une des formes de prévention situées le plus en amont est manifestement la formation des doctorants aux principes de l'éthique scientifique. L'accompagnement de leurs premiers manuscrits, que ce soit par leurs encadrants, les coauteurs ou les éditeurs, offre un contexte pertinent pour assurer de manière très concrète une telle formation.

L'enjeu est de taille. Comme l'observait déjà en 1992 le sociologue canadien Serge Larivée ${ }^{1}$, auteur d'un rapport sur la fraude scientifique, « il s'en faudrait peut-être de peu, particulièrement en période de récession, pour que les payeurs de taxes, influencés par la couverture journalistique sensationnaliste de quelques cas célèbres, contestent la masse budgétaire impartie à la recherche scientifique dans tel ou tel domaine ou même dans son ensemble. »

Un peu plus de vingt ans plus tard, le contexte n'a hélas guère évolué favorablement. Notre vigilance à l'égard du respect de l'éthique éditoriale demeure plus que jamais nécessaire.

Jacques TASSIN

Rédacteur en chef

${ }^{1}$ Larivée S., Baruffaldi M., 1992. Les Fraudes scientifiques. Rapport préliminaire. Université de Montréal, Conseil de recherche en sciences humaines du Canada, 239 p. 